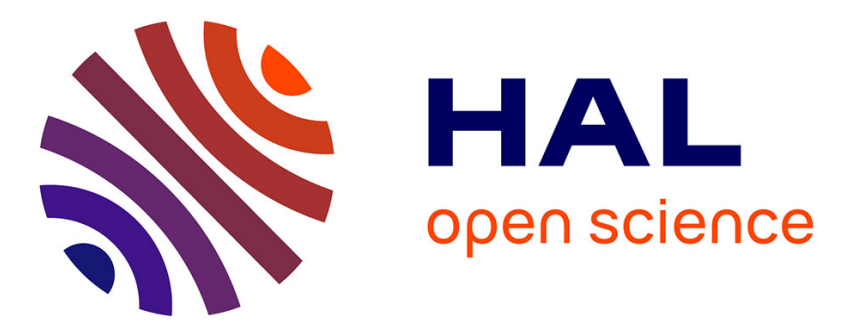

\title{
Modelling chemical composition in electric systems - implications to the dynamics of dye-sensitised solar cells
}

\author{
T. Kovanen, T. Tarhasaari, L. Kettunen, J. Korppi-Tommola
}

\section{To cite this version:}

T. Kovanen, T. Tarhasaari, L. Kettunen, J. Korppi-Tommola. Modelling chemical composition in electric systems - implications to the dynamics of dye-sensitised solar cells. European Physical Journal: Applied Physics, 2010, 50 (1), pp.11101. 10.1051/epjap/2010015 . hal-00581874

\section{HAL Id: hal-00581874 \\ https://hal.science/hal-00581874}

Submitted on 1 Apr 2011

HAL is a multi-disciplinary open access archive for the deposit and dissemination of scientific research documents, whether they are published or not. The documents may come from teaching and research institutions in France or abroad, or from public or private research centers.
L'archive ouverte pluridisciplinaire HAL, est destinée au dépôt et à la diffusion de documents scientifiques de niveau recherche, publiés ou non, émanant des établissements d'enseignement et de recherche français ou étrangers, des laboratoires publics ou privés. 


\title{
Modelling chemical composition in electric systems - implications to the dynamics of dye-sensitised solar cells
}

\author{
T. Kovanen ${ }^{1}$ a, T. Tarhasaari ${ }^{1}$, L. Kettunen ${ }^{1}$, and J. Korppi-Tommola ${ }^{2}$ \\ 1 Tampere University of Technology, Electromagnetics, P.O.Box 692, FI-33101 Tampere, Finland \\ ${ }^{2}$ University of Jyväskylä, Department of Chemistry/Nanoscience Center, P.O.Box 35, FI-40014 Jyväskylä, Finland
}

Received: date / Revised version: date

\begin{abstract}
Classical electromagnetism provides limited means to model electric generators. To extend the classical theory in this respect, additional information on microscopic processes is required. In semiconductor devices and electrochemical generators such information may be obtained by modelling chemical composition. Here we use this approach for the modelling of dye-sensitised solar cells. We simulate the steady-state current-voltage characteristics of such a cell, as well as its transient response. Dynamic simulations show optoelectronic hysteresis in these cells under transient light pulse illumination.
\end{abstract}

PACS. 82.47 Applied electrochemistry - 72.40 Photoconduction and photovoltaic effects - 73.63 Electronic transport in nanoscale materials and structures

\section{Introduction}

In certain physical systems the main process is the generation of electric power by chemical reactions. By itself, the classical (phenomenological) electromagnetic theory provides limited means to the modelling of these systems. Consequently, they have drawn a lot of attention from the physics and chemistry research communities, and addi-

a E-mail: tuomas.kovanen@tut.fi, Fax: +358331152160 tional information on microscopic processes has been combined with the classical theory for a more detailed model.

When employing the classical theory, generation and dissipation are usually confined to distinct parts of space. In the dissipative part, the electric field intensity $e$ of a given charge distribution is determined by the Poisson equation of electrostatics together with the charge conservation law and the classical Ohm's law. In the generator part of space, on the other hand, the product of $\boldsymbol{e}$ and the current density $j$ needs to be made negative. To 
accomplish this, the Ohm's law could be modified by an additional source current term assumed to be known in advance.

For some purposes, a more detailed model than provided by the classical theory can be useful. Such a model necessarily involves an alternative for Ohm's law to be used in the description of current generation. In addition to the current density, also charge density $\rho$ is not known in advance but need to be obtained as a part of the problem solution. Here we employ the modelling of chemical composition for the above mentioned tasks. Since we have been unable to find a treatment where the physicalchemical setting is introduced in logical order, a detailed formalisation is given in the first part of the paper.

Examples in which the classical approach is usually adjusted in this way include semiconductor devices and various types of solar cells [1-6]. A particular example is a dye-sensitised solar cell in which generation occurs throughout a film of macroscopic thickness $[7,8]$. In the latter part of the paper, we demostrate the method by generating a model for dye-sensitised solar cells. From the point of view of these cells, we focus here on their dynamic behaviour under transient light pulse illumination.

\section{Formalisation}

Let us consider a physical system as being composed of different subsystems. In constructing and superposing these subsystems, quantities for which addition and scalar multiplication make physical sense are of particular importance for modelling purposes. These two elementary oper- ations open the way to efficiently employ mathematics in a modelling process, that is, in the translation of physical assumptions and laws into form accessible by experimentation. The value of mathematics is thus in assisting us with practical problems, and, on the other hand, a request for such assistance makes its use indispensable.

\subsection{The chemical subsystem}

To describe chemical composition in the spatial domain, we assume the precence of appropriate energy carriers called substances. These can be, for instance, certain molecules, electrons or photons. By composition we refer to quantities that indicate amounts of substances per unit volume at each mesoscopic point of space. ${ }^{1}$ Formally, a proper quantity for each element $s$ of a set of substances is the substance density $n_{s}$, see Appendix A for details. Since each point of space can be considered as an open system, transport of substances exists. In addition, substances are generated and regenerated locally by chemical reactions. Proper quantities to take these processes into account are substance fluxes $\boldsymbol{j}_{s}$ and generation rates $r_{s}$. Also, energy is stored and released in the spatial domain,

\footnotetext{
1 At each mesoscopic point of space there is statistical abundance of quanta of energy carriers for their density to be a meaningful concept. Also, the length scale of the largest spatial inhomogenities smoothed out in the model determines the smallest length scale under explicit consideration. Length scales much smaller than this limit are then referred microscopic. Unless otherwise stated, all points of space referred in this paper are mesoscopic.
} 
and quantities that characterise the response of the free energy density to changes in substance densities will be of crucial importance. Such quantities are named with hindsight as electrochemical potentials $\mu_{s}$, the detailed definition of which is given in Appendix B.

To obtain starting points (axioms) for modelling, physical and chemical knowledge of relationships between these quantities is utilised. Such laws serve as predicates for the quantities and may be used to define relations between them, see Appendix A for details. A conjunction of carefully chosen laws (expressed as the intersection of relations) will then uniquely determine these quantities. A type of conservation law is that the substance flowing out of each space point per unit time must be generated at the point or the substance density at this point must change accordingly. This idea is employed in the model by introducing continuity relations

$$
\left\{\left(n_{s}, r_{s}, \boldsymbol{j}_{s}\right): \operatorname{div} \boldsymbol{j}_{s}+\partial_{t} n_{s}=r_{s}\right\}
$$

for each substance $s$. In addition, we need to express the behaviour of background materials in an appropriate way. Relying on the second law of thermodynamics we require that substance is transported to the direction of lower electrochemical potential until equilibrium is reached [9-11]. This is done by introducing pointwise mappings $L_{s}$, such that $\boldsymbol{j}_{s}=-L_{s}\left(\operatorname{grad} \mu_{s}\right)$. Using these mappings, the idea can be employed by introducing relations

$$
\left\{\left(\mu_{s}, \boldsymbol{j}_{s}\right): \boldsymbol{j}_{s}=-L_{s}\left(\operatorname{grad} \mu_{s}\right)\right\}
$$

for each substance $s$. Denoting the array of all substance densities as $n_{\mathcal{S}}$, we express the dependence of generation rates on composition by introducing pointwise mappings

$F_{s}$, such that $r_{s}=F_{s}\left(n_{\mathcal{S}}\right)$, and further relations

$$
\left\{\left(n_{\mathcal{S}}, r_{s}\right): r_{s}=F_{s}\left(n_{\mathcal{S}}\right)\right\}
$$

which are referred in chemistry as rate laws [11]. To obtain the substance densities as a part of the solution, we would still need relations between $\mu_{s}$ 's and $n_{s}$ 's. This need will drive us to decompose electrochemical potentials into two parts. For this, we turn our focus to the electric subsystem.

\subsection{Connection to the electric subsystem}

Informally, the charge density $\rho$ is a sum of contributions by different charged substances. To make use of the idea, we introduce relation

$$
\left\{\left(n_{\mathcal{S}}, \rho\right): \rho=\sum_{s} q_{s} n_{s}\right\}
$$

where each coefficient $q_{s}$ is the charge per unit amount of substance $s$. The same idea applies for the current density $j$. Therefore, we introduce relation

$$
\left\{\left(\boldsymbol{j}_{\mathcal{S}}, \boldsymbol{j}\right): \boldsymbol{j}=\sum_{s} q_{s} \boldsymbol{j}_{s}\right\}
$$

By considering (1), (4) and (5) together, it can be seen that the charge conservation $\operatorname{div} \boldsymbol{j}+\partial_{t} \rho=0$ further implies a relation for generation rates. That is, we have

$$
\left\{\left(r_{\mathcal{S}}\right): \sum_{s} q_{s} r_{s}=0\right\}
$$

which is imposed in the construction of the rate laws.

Let us next introduce quantities that characterise the response of the electric energy density to changes in substance densities. These quantities are named here substance potentials $\mu_{s}^{\mathrm{el}}$, and they are constructed in view 
of the correspondence $\mu_{s}^{\mathrm{el}}=q_{s} \phi^{\mathrm{el}}$ with the electric potential $\phi^{\mathrm{el}}$, see Appendix B for details. It is notable that the substance potentials need not coincide with the experimentally relevant electrochemical potentials introduced above. To explain this difference (as is done for electrolytes in [12]), microscopic processes are called for.

At this point we decompose the electrochemical potentials into microscopic (or chemical) and mesoscopic (or electric) parts by introducing the chemical potential of substance $s$ as the difference $\mu_{s}^{\mathrm{ch}}=\mu_{s}-\mu_{s}^{\mathrm{el}}$. Since $\mu_{s}^{\mathrm{el}}$ and $\phi^{\mathrm{el}}$ are related the electrochemical potential can be written as $\mu_{s}=\mu_{s}^{\mathrm{ch}}+q_{s} \phi^{\mathrm{el}}$. The substance fluxes are also decomposed according to the above decomposition of potentials. For this, mappings $L_{s}$ are required to be additive, and relations (2) are replaced with relations

$$
\left\{\left(\phi^{\mathrm{el}}, \mu_{s}^{\mathrm{ch}} \boldsymbol{j}_{s}\right): \boldsymbol{j}_{s}=-L_{s}\left(\operatorname{grad} \mu_{s}^{\mathrm{ch}}\right)-L_{s}\left(q_{s} \operatorname{grad} \phi^{\mathrm{el}}\right)\right\},
$$

which describe the chemical and electric influences to the individual substance fluxes in separate parts. In the above relations the mappings $L_{s}$ are further required to be odd, so the model includes the idea that zero flux is obtained by balancing the two influences, which is the content of the (generalised) Einstein relation. To express the pointwise dependence of $\mu_{s}^{\mathrm{ch}}$ 's on $n_{s}$ 's, we introduce pointwise mappings $H_{s}$, such that $\mu_{s}^{\mathrm{ch}}=H_{s}\left(n_{s}\right)$, and relations

$$
\left\{\left(n_{s}, \mu_{s}^{\mathrm{ch}}\right): \mu_{s}^{\mathrm{ch}}=H_{s}\left(n_{s}\right)\right\}
$$

for each substance $s$
By taking the intersection of (1), (3), (7) and (8), we get $^{2}$

$$
\begin{aligned}
\left\{\left(n_{\mathcal{S}}, \phi^{\mathrm{el}}\right):\right. & -\operatorname{div}\left(L_{s}\left(\operatorname{grad} H_{s}\left(n_{s}\right)\right)+L_{s}\left(q_{s} \operatorname{grad} \phi^{\mathrm{el}}\right)\right)+\partial_{t} n_{s} \\
& \left.=F_{s}\left(n_{\mathcal{S}}\right)\right\}
\end{aligned}
$$

for each substance $s$. By further taking the intersection of (4), (9) and the relation of electrostatics,

$$
\left\{\left(\rho, \phi^{\mathrm{el}}\right):-\operatorname{div}\left(\epsilon \operatorname{grad} \phi^{\mathrm{el}}\right)=\rho\right\}
$$

we obtain a unique element (up to boundary conditions) $\left(n_{\mathcal{S}}, \rho, \phi^{\mathrm{el}}\right)$ which satisfies all the requirements of the modelling. Furthermore, according to (5) and (7), the current density is given as

$$
\boldsymbol{j}=-\sum_{s} q_{s} L_{s}\left(\operatorname{grad} \mu_{s}^{\mathrm{ch}}\right)-\sum_{s} q_{s} L_{s}\left(q_{s} \operatorname{grad} \phi^{\mathrm{el}}\right),
$$

which can be interpreted as an extended Ohm's law. Relations (9) and (10) form what is called the coupled driftdiffusion-reaction problem, in which the substance diffusivity is defined as $D_{s}=L_{s} \circ \partial \mu_{s}^{\mathrm{ch}} / \partial n_{s}$ (pointwise derivative, not the Fréchet derivative of Appendix B), and the substance conductivity is defined as $\sigma_{s}=q_{s} L_{s} \circ q_{s}$.

Finally, in view of a concrete instance such as the dyesensitised solar cell, the set of substances, the chemical reactions present and the constitutive relations (3), (7) and (8) are left to be determined.

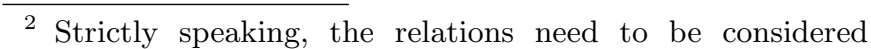
as subsets of the same set (the linear space of elements $\left(n_{\mathcal{S}}, \phi^{\mathrm{el}}, \mu_{\mathcal{S}}^{\mathrm{ch}}, r_{\mathcal{S}}, \boldsymbol{j}_{\mathcal{S}}\right)$ in this case $)$ to take the intersection. This should be kept in mind throughout this paper. 


\section{Application}

Detailed modelling of dye-sensitised solar cells (referred by dye cells below) under steady-state operation have been performed elsewhere [13]. With an eye for these results we aim to generate a model with some predictive power concerning the dynamic behaviour of the cell, and simulate the steady-state operation mainly for illustration purposes. To the authors' knowledge, such dynamic modelling have been restricted to a model that includes only one substance [14]. Alternatively, one has considered timeharmonic situations [15-20].

\subsection{Structure and operation of the dye cell}

Let us first have a brief overview of the dye cell. In this device the large surface area of a nanoporous semiconductor electrode is employed to attach a large amount of photoactive dye on the surface where the photochemical reactions take place. The structure of the cell is shown in Fig. 1. The continuous performance of the cell is made possible by the electrolyte which reduces the photoexcited dye while the electrons released to the semiconductor are transported through the nanoparticle network, the conducting substrate and the external load to the counter electrode and again back to the electrolyte [7,21].

\subsection{Relevant substances}

Keeping in mind the preceding ideas, we begin with specifying the set of substances. A typical electrolyte contains at least iodide/triiodide redox pair $\left(I^{-} / I_{3}^{-}\right)$, lithium ions
$\left(\mathrm{Li}^{+}\right)$and tert-butylpyridine $(t B P)[18,22]$. In the semiconductor nanoparticles, free electrons can be trapped to localised energy states [8]. Hence, in the active electrode region two types of electrons are of interest, trapped electrons $e_{\mathrm{t}}^{-}$and conduction electrons $e_{\mathrm{c}}^{-}$, in addition to the electrolyte species. This is not the case in the counter electrode where only conduction electrons need to be considered. Finally, by including photons $h \nu$, the relevant substances can be collected into

$$
\left\{h \nu, e_{\mathrm{c}}^{-}, e_{\mathrm{t}}^{-}, I^{-}, I_{3}^{-}, L i^{+}, t B P\right\}
$$

This selection of substances means that the dye, the electrolyte solvent and the semiconductor crystal structure are taken into account only by the constitutive relations.

Often, in modelling the dye cell, also the effect of photons, ions and molecules on the transport and generation of the modelled substances is dubbed into the constitutive relations. ${ }^{3}$ Accordingly, the resulting set of substances consists of only conduction and trapped electrons. It is typically thought that the larger the set of substances, the deeper the physical and/or chemical understanding. Here we consider conduction and trapped electrons explicitly to obtain a dynamic model for the cell.

\subsection{Chemical reactions}

Chemical reactions occur in each point of the active electrode (anode) and at the surface of the counter electrode

\footnotetext{
3 This is not justified a priori, but it requires certain special
} properties from the situation. Such properties are discussed in sections 3.4 and 3.5 . 
(cathode). The reactions may be written using chemical formalism as follows $[8,23]$ :

Anode:

$2 h \nu+3 I^{-} \rightarrow I_{3}^{-}+2 e_{\mathrm{c}}^{-}$

$e_{\mathrm{c}}^{-} \rightarrow e_{\mathrm{t}}^{-}$

$e_{\mathrm{t}}^{-} \rightarrow e_{\mathrm{c}}^{-}$

$I_{3}^{-}+2 e_{\mathrm{c}}^{-} \rightarrow 3 I^{-}$

$I_{3}^{-}+2 e_{\mathrm{t}}^{-} \rightarrow 3 I^{-}$,

$I_{3}^{-}+e_{\mathrm{c}}^{-}+e_{\mathrm{t}}^{-} \rightarrow 3 I^{-}$

Cathode: $I_{3}^{-}+2 e_{\mathrm{c}}^{-} \rightarrow 3 I^{-}$.

Here we have written the net photoreaction at the anode which does not explicitly state the precence of the dye. Notice that the cathode reaction occurs also at the anode. This recombination reaction is considered to be a significant degrading factor of the cell performance, and it largely motivates the mesoscopic modelling of the cell.

\subsection{Constitutive relations}

There is a controversy of what should be done with the constitutive relations of field theories in general. One conception defends their determination strictly by measurement to confine any uncertainty into such measurements, while others try to derive them from the fundamental assumptions of microscopic theories. In connection with dye cells both of the approaches have gained interest, and here we will also employ both of them.

\subsection{1 "Flux laws" $\left(L_{s}\right)$}

In accordance with the band theory of solids, relation (7) for conduction electrons is given as

$$
\left\{\left(\phi^{\mathrm{el}}, \mu_{e_{\mathrm{c}}^{-}}^{\mathrm{ch}}, \boldsymbol{j}_{e_{\mathrm{c}}^{-}}\right): \boldsymbol{j}_{e_{\mathrm{c}}^{-}}=-\frac{n_{e_{\mathrm{c}}^{-}} u_{e_{\mathrm{c}}^{-}}}{\left|q_{e_{\mathrm{c}}^{-}}\right|}\left(\operatorname{grad} \mu_{e_{\mathrm{c}}^{-}}^{\mathrm{ch}}+q_{e_{\mathrm{c}}^{-}} \operatorname{grad} \phi^{\mathrm{el}}\right)\right\}
$$

where $u_{e_{\mathrm{c}}^{-}}$is the mobility [24], which holds information on the microstructure of each mesoscopic point of Fig. 1. Here we have identified $\mu_{e_{\mathrm{c}}^{-}}$with the difference of the quasiFermi level of electrons and the Fermi-level at equilibrium. The trap states are localised in space so for trapped electrons the mobility is zero. Therefore, the trapped electron flux vanishes so relation (7) is

$$
\left\{\left(\phi^{\mathrm{el}}, \mu_{e_{\mathrm{t}}^{-}}^{\mathrm{ch}}, \boldsymbol{j}_{e_{\mathrm{t}}^{-}}\right): \boldsymbol{j}_{e_{\mathrm{t}}^{-}}=\mathbf{0}\right\}
$$

3.4.2 "Potential laws" $\left(H_{s}\right)$

By treating conduction electrons as a non-degenerate (classical) gas [24], relation (8) for conduction electrons is given as

$$
\left\{\left(n_{e_{\mathrm{c}}^{-}}, \mu_{e_{\mathrm{c}}^{-}}^{\mathrm{ch}}\right): \mu_{e_{\mathrm{c}}^{-}}^{\mathrm{ch}}=k_{\mathrm{B}} T \ln \left(\frac{n_{e_{\mathrm{c}}^{-}}}{n_{\mathrm{c}}^{\mathrm{eq}}}\right)\right\}
$$

where $n_{\mathrm{c}}^{\mathrm{eq}}$ is the equilibrium conduction electron density in the dark, $k_{\mathrm{B}}$ is Boltzmann's constant, and $T$ is temperature. Here we have further identified $q_{e_{c}^{-}} \phi^{\text {el }}$ with the deviation of the conduction band edge from its equilibrium level. As a consequence of the predicate in relation (14), the relation (8) for trapped electrons will not be needed. 


\subsection{3 "Rate laws" $\left(F_{s}\right)$}

The rate law of generation of conduction electrons is composed of contributions of the different reactions at the anode. That is, we have

$$
\begin{aligned}
\left\{\left(n_{h \nu}, n_{e_{\mathrm{c}}^{-}}, n_{e_{\mathrm{t}}^{-}}, r_{e_{\mathrm{c}}^{-}}\right): r_{e_{\mathrm{c}}^{-}}=\right. & F_{e_{\mathrm{c}}^{-}}^{\mathrm{p}}\left(n_{h \nu}\right) \\
& \left.+F_{e_{\mathrm{c}}^{-}}^{\mathrm{t}}\left(n_{e_{\mathrm{c}}^{-}}, n_{e_{\mathrm{t}}^{-}}\right)+F_{e_{\mathrm{c}}^{-}}^{\mathrm{cb}}\left(n_{e_{\mathrm{c}}^{-}}\right)\right\},
\end{aligned}
$$

where the generation rate of conduction electrons by photons is

$$
F_{e_{\mathrm{c}}^{-}}^{\mathrm{p}}\left(n_{h \nu}\right)=-F_{h \nu}\left(n_{h \nu}\right)
$$

and the generation rate of conduction electrons by trapping and detrapping can be given as

$$
F_{e_{\mathrm{c}}^{-}}^{\mathrm{t}}\left(n_{e_{\mathrm{c}}^{-}}, n_{e_{\mathrm{t}}^{-}}\right)=k_{\text {detrap }} n_{e_{\mathrm{t}}^{-}}-k_{\text {trap }}\left(1-\frac{n_{e_{\mathrm{t}}^{-}}}{N_{\text {trap }}}\right) n_{e_{\mathrm{c}}^{-}},
$$

where $k_{\text {detrap }}$ is the rate constant of detrapping, $k_{\text {trap }}$ is the rate constant of trapping, and $N_{\text {trap }}$ is the density of traps $[15,19]$. Equation (18) is a simplification of theories where the trapped electrons are distributed in energy $[26,27]$. To employ such theories it would be necessary to determine the trapped electron density (per unit volume and energy) also in "energy space". Finally, the generation rate of conduction electrons by recombination (negative quantity) is taken as first order in excess conduction electron density $[15,20]$. That is

$$
F_{e_{\mathrm{c}}^{-}}^{\mathrm{cb}}\left(n_{e_{\mathrm{c}}^{-}}\right)=-k_{\mathrm{cb}}\left(n_{e_{\mathrm{c}}^{-}}-n_{\mathrm{c}}^{\mathrm{eq}}\right),
$$

where $k_{\mathrm{cb}}$ is the rate constant of recombination from the conduction band. The above rate law is a simplification of experimental evidence suggesting that recombination is second order in electron density [18]. To obtain an expression for $F_{e_{\mathrm{c}}^{\mathrm{p}}}^{\mathrm{p}}\left(n_{h \nu}\right)$ in (16), the generation rate of photons (negative quantity) $F_{h \nu}\left(n_{h \nu}\right)$ appearing on the right hand side of (17) is usually given a priori as a function of position and time. This corresponds to the assumption that the photon density is independent of the densities of other substances in the cell.

For the rate law of generation of trapped electrons, we have

$$
\left\{\left(n_{e_{\mathrm{c}}^{-}}, n_{e_{\overline{\mathrm{t}}}}, r_{e_{\mathrm{t}}^{-}}\right): r_{e_{\mathrm{t}}^{-}}=F_{e_{\mathrm{t}}^{-}}^{\mathrm{t}}\left(n_{e_{\mathrm{c}}^{-}}, n_{e_{\overline{\mathrm{t}}}^{-}}\right)+F_{e_{\mathrm{t}}^{-}}^{\mathrm{tb}}\left(n_{e_{\mathrm{t}}^{-}}\right)\right\},
$$

where the generation rate of trapped electrons by trapping and detrapping is

$$
F_{e_{\mathrm{t}}^{-}}^{\mathrm{t}}\left(n_{e_{\mathrm{c}}^{-}}, n_{e_{\mathrm{t}}^{-}}\right)=-F_{e_{\mathrm{c}}^{-}}^{\mathrm{t}}\left(n_{e_{\mathrm{c}}^{-}}, n_{e_{\overline{\mathrm{t}}}^{-}}\right)
$$

and the generation rate of trapped electrons by recombination with electrolyte (negative quantity) is taken as first order in excess trapped electron density [28], that is

$$
F_{e_{\mathrm{t}}^{-}}^{\mathrm{tb}}\left(n_{e_{\mathrm{t}}^{-}}\right)=-k_{\mathrm{tb}}\left(n_{e_{\mathrm{t}}^{-}}-n_{\mathrm{t}}^{\mathrm{eq}}\right),
$$

where $k_{\mathrm{tb}}$ is the rate constant of recombination of the trapped electrons, and $n_{\mathrm{t}}^{\mathrm{eq}}$ is the equilibrium trapped electron density in the dark.

Finally, we emphasise that the present formulation of the rate laws excludes the effect of the density variations of ions and molecules on the generation rates. The use of such rate laws is justified here due to the large density of electolyte species which is only slightly perturbed under cell operation according to earlier modelling results [13]. 


\subsection{Formulation}

The axioms necessary for mathematical modelling have now been collected into relations (1), (13), (14), (15), (16) and (20). By taking the intersection of these relations, we get the coupled drift-diffusion problem with trapping and recombination expressed by relations

$$
\begin{aligned}
& \left\{\left(n_{e_{\mathrm{c}}^{-}}, n_{e_{\mathrm{t}}^{-}}, \phi^{\mathrm{el}}\right):-\operatorname{div}\left(D_{e_{\mathrm{c}}^{-}} \operatorname{grad} n_{e_{\mathrm{c}}^{-}}\right.\right. \\
& \left.\quad+n_{e_{\mathrm{c}}^{-}} u_{e_{\mathrm{c}}^{-}} \operatorname{grad} \phi^{\mathrm{el}}\right)+\partial_{t} n_{e_{\mathrm{c}}^{-}}=-F_{h \nu}\left(n_{h \nu}\right)+k_{\mathrm{detrap}} n_{e_{\mathrm{t}}^{-}} \\
& \left.\quad-k_{\text {trap }}\left(1-\frac{n_{e_{\mathrm{t}}^{-}}}{N_{\mathrm{trap}}}\right) n_{e_{\mathrm{c}}^{-}}-k_{\mathrm{cb}}\left(n_{e_{\mathrm{c}}^{-}}-n_{\mathrm{c}}^{\mathrm{eq}}\right)\right\}
\end{aligned}
$$

and

$$
\begin{aligned}
& \left\{\left(n_{e_{\mathrm{c}}^{-}}, n_{e_{\mathrm{t}}^{-}}\right): \partial_{t} n_{e_{\mathrm{t}}^{-}}=-k_{\text {detrap }} n_{e_{\mathrm{t}}^{-}}+k_{\text {trap }}\left(1-\frac{n_{e_{\mathrm{t}}^{-}}}{N_{\mathrm{trap}}}\right) n_{e_{\mathrm{c}}^{-}}\right. \\
& \left.\quad-k_{\mathrm{tb}}\left(n_{e_{\mathrm{t}}^{-}}-n_{\mathrm{t}}^{\mathrm{eq}}\right)\right\}
\end{aligned}
$$

and further relation

$$
\begin{aligned}
& \left\{\left(n_{e_{\mathrm{c}}^{-}}, n_{e_{\mathrm{t}}^{-}}, n_{I^{-}}, n_{I_{3}^{-}}, n_{L i^{+}}, \phi^{\mathrm{el}}\right):-\operatorname{div}\left(\epsilon \operatorname{grad} \phi^{\mathrm{el}}\right)\right. \\
& \left.\quad=q_{e_{\mathrm{c}}^{-}} n_{e_{\mathrm{c}}^{-}}+q_{e_{\mathrm{t}}^{-}} n_{e_{\mathrm{t}}^{-}}+\sum_{s \in\left\{I^{-}, I_{3}^{-}, L i^{+}\right\}} q_{s} n_{s}\right\} .
\end{aligned}
$$

Notice that although the ions are not considered explicitly in the chosen modelling they need to be taken into account in (4), and hence in (25). Clearly, we have taken a shortcut when leaving them out of the model. However, this was done with the knowledge of an earlier modelling result [13]. According to this result, the local charge neutrality is maintained (to a good approximation) in the active electrode under cell operation, corresponding to a vanishing electric field according to relation (25). Relying on this result, we leave out relation (25) and the electric field term in (23).

\subsection{Current and voltage}

We need the global electric quantities, the cell current and the cell voltage, in terms of conduction electron density. As stated in Appendix $\mathrm{C}$, the problem is reduced to one dimension, and the spatial domain consisting of the active electrode is parametrised by $x \in[0, d]$, such that $x=0$ is the substrate interface and $x=d$ is the electrolyte interface of the active electrode. As a result, the cell current (per unit area) $I$ is given directly by (5), (13) and (15) as

$$
\begin{aligned}
& \left.I(t)=-\boldsymbol{j}(0, t) \cdot \boldsymbol{n}(0)=-\sum_{s \in\left\{e_{c}^{-}, I^{-}, I_{3}^{-}, L i\right.}{ }_{s}\right\} \\
& =-q_{e_{\mathrm{c}}^{-}} D_{e_{\mathrm{c}}^{-}} \frac{\partial n_{e_{\mathrm{c}}^{-}}(x, t)}{\partial x}{ }_{\mid x=0},
\end{aligned}
$$

since the current due to the ions vanish at $x=0$. The cell voltage, on the other hand, needs some argumentation. Let us assume for a while that the counter electrode is part of the spatial domain. Then, excluding the ohmic voltage drop caused by the conducting substrate, the (positive) cell voltage $V$ is given as

$$
V(t)=\frac{\mu_{e_{\mathrm{c}}^{-}}(\text {counter electrode }, t)}{q_{e_{\mathrm{c}}^{-}}}-\frac{\mu_{e_{\mathrm{c}}^{-}}(0, t)}{q_{e_{\mathrm{c}}^{-}}} .
$$

Typically, it is assumed that the counter electrode is in its equilibrium state of the dark, so that the electrochemical potential vanishes there. This leads to the widely employed expression

$$
V(t)=-\frac{\mu_{e_{\mathrm{c}}^{-}}(0, t)}{q_{e_{\mathrm{c}}^{-}}}=-\frac{k_{\mathrm{B}} T}{q_{e_{\mathrm{c}}^{-}}} \ln \left(\frac{n_{e_{\mathrm{c}}^{-}}(0, t)}{n_{\mathrm{c}}^{\mathrm{eq}}(0)}\right),
$$

where the latter equality follows from relation (15) with the assumption that $\mu_{e_{\mathrm{c}}^{-}}=\mu_{e_{\mathrm{c}}^{-}}^{\mathrm{ch}}$. 


\subsection{Boundary conditions}

To specify the electron densities uniquely, boundary and initial conditions are still required. For clarification we review here three types of conditions. To begin with, the current-voltage characteristics of the cell - the macroscopic counterpart of (11) - is obtained for steady state by successively adjusting the cell voltage through the voltage range of interest and solving for the current. As the cell voltage is related to conduction electron density by (28) this can be done by introducing relation

$\left\{n_{e_{\mathrm{c}}^{-}}: n_{e_{\mathrm{c}}^{-}}(0)=n_{\mathrm{c}}^{\mathrm{eq}}(0) e^{-q_{e_{\mathrm{c}}^{-}} V /\left(k_{\mathrm{B}} T\right)}\right.$ and $\left.\left.\frac{\mathrm{d} n_{e_{\mathrm{c}}^{-}}(x)}{\mathrm{d} x}\right|_{\mid x=d}=0\right\}$,

where the latter part of the predicate expresses the zero electron flux to the electrolyte. The procedure of finding the unique element of the intersection of (23), (24) and (29) is given in Appendix $\mathrm{C}$ for light illumination from the substrate side of the cell. The current-voltage curves thus obtained are given in the results section.

The above method, however, cannot be used in the modelling of transient responses with a fixed load resistance since the voltage corresponding to the fixed resistance depends on light intensity (and possibly on the history of light intensity), and it cannot be known in advance. To model transient responses with an arbitrary load resistance $R$, the Ohm's law $V=R I$ need to be imposed. This corresponds to a nonlinear boundary condition, and it results in relation

$$
\begin{aligned}
& \left\{\left(n_{e_{\mathrm{c}}^{-}}, n_{e_{\mathrm{t}}^{-}}\right):-\frac{k_{\mathrm{B}} T}{q_{e_{\mathrm{c}}^{-}}} \ln \left(\frac{n_{e_{\mathrm{c}}^{-}}(0, \cdot)}{n_{\mathrm{c}}^{\mathrm{eq}}(0)}\right)=-\left.R q_{e_{\mathrm{c}}^{-}} D_{e_{\mathrm{c}}^{-}} \frac{\partial n_{e_{\mathrm{c}}^{-}}(x, \cdot)}{\partial x}\right|_{\mid x=0},\right. \\
& {\frac{\partial n_{e_{\mathrm{c}}^{-}}(x, \cdot)}{\partial x}}_{\mid x=d}=0, \quad n_{e_{\mathrm{c}}^{-}}(\cdot, 0)=n_{\mathrm{c}}^{\mathrm{ref}}, \\
& \text { and } \left.n_{e_{\mathrm{t}}^{-}}(\cdot, 0)=n_{\mathrm{t}}^{\mathrm{ref}}\right\} \text {, }
\end{aligned}
$$

where $n_{\mathrm{c}}^{\text {ref }}$ and $n_{\mathrm{t}}^{\text {ref }}$ specify the initial condition to a reference steady state.

We perform transient simulations only for the shortcircuit current and open-circuit voltage. Both situations can be realised by using the kinetic rate constant of electron extraction $k_{\text {ext }}$. Accordingly, we introduce relation

$$
\begin{aligned}
& \left\{\left(n_{e_{\mathrm{c}}^{-}}, n_{e_{\mathrm{t}}^{-}}\right):\left.D_{\mathrm{c}} \frac{\partial n_{\mathrm{c}}(x, \cdot)}{\partial x}\right|_{\mid x=0}=k_{\mathrm{ext}}\left(n_{\mathrm{c}}(0, \cdot)-n_{\mathrm{c}}^{\mathrm{eq}}(0)\right),\right. \\
& \left.{\frac{\partial n_{e_{\mathrm{c}}^{-}}(x, \cdot)}{\partial x}}_{\mid x=d}=0, \quad n_{e_{\mathrm{c}}^{-}}(\cdot, 0)=n_{\mathrm{c}}^{\mathrm{ref}} \text { and } n_{e_{\mathrm{t}}^{-}}(\cdot, 0)=n_{\mathrm{t}}^{\mathrm{ref}}\right\} .
\end{aligned}
$$

The open-circuit case when negligible amount of electrons is extracted to the circuits is obtained by setting $k_{\text {ext }}=$ 0 , and the short-circuit situation when $n_{\mathrm{c}}(0)=n_{\mathrm{c}}^{\mathrm{eq}}(0)$ is (formally) obtained in the limit $k_{\text {ext }}=\infty$. It is notable that a finite resistance situation cannot be modelled by a finite value of $k_{\text {ext }}$ since this would contradict the Ohm's law $V=R I$. The method to take the intersection of (23), (24) and (31) is given in Appendix C.

\subsection{Parameter values}

The parameter values of the constitutive relations used in the modelling were taken from literature and are listed in Table 1. The order of magnitude values for the first order recombination rate constants, as well as for the rate constants of trapping and detrapping, were estimated based 
Table 1. Parameter values used in the modelling, and their sources.

\begin{tabular}{lcr}
\hline parameter & value & source \\
\hline$d$ & $10^{-5} m$ & {$[18]$} \\
$T$ & $300 K$ & {$[19]$} \\
$D_{\mathrm{c}}$ & $10^{-6} \mathrm{~m}^{2} \mathrm{~s}^{-1}$ & {$[19]$} \\
$k_{\mathrm{cb}}$ & $10 \mathrm{~s}^{-1}$ & estimated $[18,19]$ \\
$k_{\mathrm{tb}}$ & $10 \mathrm{~s}^{-1}$ & estimated $[18,19]$ \\
$k_{\text {trap }}$ & $10^{7} \mathrm{~s}^{-1}$ & estimated $[18,19]$ \\
$k_{\mathrm{detrap}}$ & $10^{6} \mathrm{~s}^{-1}$ & estimated $[18,19]$ \\
$\alpha$ & $2.5 \cdot 10^{5} m^{-1}$ & {$[18]$} \\
$N_{\text {trap }}$ & $2.3 \cdot 10^{24} m^{-3}$ & {$[19]$} \\
$n_{\mathrm{c}}^{\text {eq }}$ & $10^{11} \mathrm{~m}^{-3}$ & {$[19]$} \\
\hline
\end{tabular}

on references $[18,19]$, and further adjusted to obtain typical steady-state current-voltage characteristics for various light intensities. Other parameter values were taken directly from the references indicated.

\subsection{Results}

Simulated steady-state current-voltage characteristics are shown in Fig. 2 for two different light intensities. As the curves are typical for these cells [13], we may hope for that also dynamic simulations show reasonable results. We focus on two dynamic relations predicted by the model, one between the light intensity $I_{\mathrm{h} \nu}$ and the short-circuit current $I_{\mathrm{SC}}$, and the other between $I_{\mathrm{h} \nu}$ and the open-circuit voltage $V_{\mathrm{OC}}$. Fig. 3 and Fig. 4 show the simulation results for short-circuit and open-circuit situations under a transient light pulse of five milliseconds in duration. It is clearly shown that the electric quantities lag the light exitation under such short light pulses. This is in contrast to the normal operating conditions of a solar cell under which the cell performance can be considered as successive steady states.

The fast transient simulations under short-circuit conditions elucidate the cell performance under the effect trapping-detrapping process. Typically, the process is taken into account only partly by the use of an "effective" diffusion coefficient (see, for instance, [31]). It is known that a dynamic model involving only conduction electrons is restricted to such a quasistatic regime under which the effective diffusion coefficient is sufficient to describe the cell dynamics [31]. The model presented here allows the simulation of the time evolution of short-circuit current and open-circuit voltage even beyond the quasistatic regime.

With the parameter values used, the short-circuit hysteresis behaviour is clearly visible when the cell is illuminated with light pulses of duration of milliseconds or shorter. It was noticed that at short-circuit conditions the detrapping rate constant in particular is a sensitive parameter that controls the extent of hysteresis. For instance, if the rate constant is reduced by two orders of magnitude then to observe hysteresis light pulses of a few hundreds of milliseconds are needed.

Even though the trapping-detrapping process need not be included explicitly in the model to describe the cell dynamics at typical working conditions of a solar cell, it may be important to study its role in the hysteresis effect. Especially, to describe the cell dynamics beyond the quasistatic 
regime a detailed account of the process is required. In particular, this may be necessary in other devices that use similar hybrid material structures, and where reproducible and fast transient behaviour is of importance. For instance, hysteresis behaviour of a $\mathrm{TiO}_{2}$-dye-electrolyte based photoconductor has been observed recently [32].

\section{Conclusions}

Limitations in using classical electromagnetism in modelling local generation of electric power can be circumvented by considering chemical composition in detail. The modelling of chemical composition allows one to incorporate information on microscopic processes in the classical description. This can be done by decomposing the influence quantity (electrochemical potential) into microscopic (labeled chemical) and mesoscopic (labeled electric) parts. A key step in the approach is to establish the constitutive relations of the chemical subsystem - this step essentially determines the uncertainty of modelling.

The dye-sensitised solar cell appears as an interesting example in which the microscopic part of the influence quantity seems to play the dominant role. In this connection, we point out assumptions that are often made to construct a model for the cell. To solve the steadystate and dynamic boundary value problems, we employ spaces of square integrable functions in methods based on finite elements. Steady-state simulations resulted in current-voltage characteristics closely resembling those typically obtained in experiments. Dynamic simulations were performed to evaluate open-circuit voltage and short-circuit ear space of appropriate functions. That is, one can add current dependence on the illumination intensity of the cell under a transient light pulse illumination. This is the first time that a model is presented that is capable of describing the time evolution of these quantities under the full influence of trapping and detrapping of electrons. A question is raised on the role of this electronic process in the optoelectronic hysteresis effect observed under fast light pulse transients.

\section{Acknowledgement}

Scholarship (TK) from FinNano research program of the Academy of Finland under FUNANO consortium (contract No 118040) is greatfully acknowledged. T.K. acknowledges Finnish Cultural Foundation for additional financial support.

\section{Appendix A: Mathematical background}

Physical quantities, like the substance density, are considered as functions in space and time. For instance, by considering the space and time as a cartesian product of $\Omega$ and $\Gamma$, the substance density is denoted as function $n_{s}: \Omega \times \Gamma \rightarrow \mathbb{R}$, whereas the image of a point $(x, t)$ by $n_{s}$ would be called $n_{s}(x, t)$. In many cases, the set $\Omega$ can be considered as a subset of the three dimensional Euclidean space, and $\Gamma$ is the closed interval $[0, T]$ of $\mathbb{R}$. By recalling the idea of addition and multiplication mentioned earlier, a prerequisite for the physical quantities considered in this paper is that each quantity belongs to the (abstract) lin- 
functions of the same space and multiply them by a scalar to obtain a new function of this space.

Relations between physical quantities are subspaces of cartesian products of the corresponding function spaces. As an example, if quantities $a$ and $b$ are functions of the spaces $A$ and $B$, and $F$ is a mapping from $A$ to $B$, a physical law $b=F(a)$ serves as a predicate for $a$ and $b$, and it defines a relation $R=\{(a, b) \in A \times B: b=F(a)\}$ (a subspace of $A \times B$ ). In this paper, we denote this as $\{(a, b): \quad b=F(a)\}$ for short. A conjuction of two laws is expressed as the intersection of the two relations $R_{1}$ and $R_{2}$ defined by the laws, that is $R_{1} \cap R_{2}$.

\section{Appendix B: Definitions}

For the definition of the electrochemical potential, we first consider the density of free energy at each point in space and time, $g(x, t)$, as a function of composition. ${ }^{4}$ Electrochemical potentials are constructed to express the response of the free energy density to variations in substance densitites. Since substance density at a point $y$ can affect to the free energy density at another point $x$ (Coulomb interactions are included), the electrochemical potential $\mu_{s}$ is defined for each substance $s$ at each point $(x, t)$ as the "derivative of $g(x, t)$ at composition $n_{\mathcal{S}}$ to the direction $n_{s} "$, and is denoted as $\partial_{n_{\mathcal{S}}} g\left(n_{s}\right)$ (the argument $(x, t)$ is dropped for notational simplicity). The operator $\partial_{n_{\mathcal{S}}} g$

\footnotetext{
${ }^{4}$ To make a connection to the thermal subsystem, we identify this energy with the (electrochemical) Gibbs free energy by assuming constant temperature and pressure [9]. Hence, all non-thermal and non-mechanical energy exchange is included.
}

(bounded and linear) is the Fréchet derivative at $n_{\mathcal{S}}$, defined by

$$
\lim _{h \rightarrow 0} \frac{\left|g\left(n_{\mathcal{S}}+h\right)-g\left(n_{\mathcal{S}}\right)-\partial_{n_{\mathcal{S}}} g(h)\right|}{\|h\|}=0,
$$

where $\|\cdot\|$ is a norm given for the substance densities.

It is noticed that the potentials constructed in this way need not be continuous in space. However, they are usually required to be continuous in certain parts of space called regularity regions. In practice, these regions are specified a priori based on the qualitative understanding of the situation. In this paper, the derivatives are restricted to the regularity regions of the potential in question.

For the definition of the substance potential, the electric energy density $g^{\mathrm{el}}$ is considered. Parallelling the construction above, substance potentials are defined as $\mu_{s}^{\mathrm{el}}=$ $\partial_{n_{\mathcal{S}}} g^{\mathrm{el}}\left(n_{s}\right)$. For the correspondence of $\mu_{s}^{\mathrm{el}}$ and the electric potential $\phi^{\mathrm{el}}$, we express (4) by a linear pointwise mapping $\hat{q}_{\mathcal{S}}$, such that $\rho=\hat{q}_{\mathcal{S}}\left(n_{\mathcal{S}}\right)$, and we further give $g^{\mathrm{el}}$ as a composition mapping $g^{\mathrm{el}}=v^{\mathrm{el}} \circ \hat{q}_{\mathcal{S}}$ by using a new mapping $v^{\mathrm{el}}$. Then, by the chain rule, it follows that

$$
\begin{aligned}
\mu_{s}^{\mathrm{el}} & =\partial_{n_{\mathcal{S}}} g^{\mathrm{el}}\left(n_{s}\right) \\
& =\partial_{\rho} v^{\mathrm{el}}(\rho) \frac{\partial \hat{q}_{\mathcal{S}}}{\partial n_{s}}\left(n_{\mathcal{S}}\right) \\
& =\phi^{\mathrm{el}} q_{s}
\end{aligned}
$$

for each substance $s$ such that $q_{s} \neq 0$, where the latter derivative is taken pointwisely.

\section{Appendix C: Solution procedures}

The structure of the dye cell has enough symmetry to reduce the problem to one spatial dimension. We parametrise 
the resulting spatial domain by $x \in[0, d]$, such that $x=0$ is the substrate interface and $x=d$ is the electrolyte interface of the active electrode. We further consider illumination from the substrate side of the cell to give the driving term for the problem using photon flux at the boundary, $I_{h \nu}(t)=-\boldsymbol{j}_{h \nu}(0, t) \cdot \boldsymbol{n}(0)$ for all $t \in[0, T]$, as

$$
-F_{h \nu}\left(n_{h \nu}\right)=g I_{h \nu}
$$

where $g$ describes the a priori fixed spatial variation. The function $g$ is typically given using the absorption coefficient $\alpha$ as $g(x)=\alpha e^{-\alpha x}[15,19]$. Before proceeding, we clarify our notation somewhat. That is, we set

$$
\begin{aligned}
& n_{\mathrm{c}}=n_{e_{\mathrm{c}}^{-}}, \\
& n_{\mathrm{t}}=n_{e_{\mathrm{t}}^{-}}, \\
& D=D_{e_{\mathrm{c}}^{-}}, \\
& N=n_{\mathrm{c}}^{\mathrm{eq}}(0) e^{-q_{e_{\mathrm{c}}^{-}} V /\left(k_{\mathrm{B}} T\right)}, \\
& c=k_{\text {trap }}\left(1-\frac{n_{\mathrm{t}}}{N_{\mathrm{trap}}}\right)+k_{\mathrm{cb}}, \\
& f=g I_{h \nu}+k_{\text {detrap }} n_{\mathrm{t}}+k_{\mathrm{cb}} n_{\mathrm{c}}^{\mathrm{eq}} .
\end{aligned}
$$

\section{Steady-state situation}

With the above notation, for a given $n_{\mathrm{t}}$, the conduction electron density $n_{\mathrm{c}}$ that belongs to the intersection of (23), (24) and (29) satisfies

$$
\begin{aligned}
-\frac{\mathrm{d}}{\mathrm{d} x} D \frac{\mathrm{d} n_{\mathrm{c}}}{\mathrm{d} x}+c n_{\mathrm{c}} & =f & & \text { in }(0, d), \\
n_{\mathrm{c}} & =N & & \text { on }\{0\}, \\
\frac{\mathrm{d} n_{\mathrm{c}}}{\mathrm{d} x} & =0 & & \text { on }\{d\} .
\end{aligned}
$$

For $n_{\mathrm{c}}$ given, on the other hand, the trapped electron density of the intersection satisfies

$$
\begin{aligned}
-k_{\text {detrap }} n_{\mathrm{t}} & +k_{\text {trap }}\left(1-\frac{n_{\mathrm{t}}}{N_{\text {trap }}}\right) n_{\mathrm{c}} \\
& -k_{\mathrm{tb}}\left(n_{\mathrm{t}}-n_{\mathrm{t}}^{\mathrm{eq}}\right)=0 \quad \text { in }[0, d] .
\end{aligned}
$$

To solve the combined problem of (C.8) and (C.9) we employ the finite element method and a fixed point iteration procedure to solve the nonlinearity caused by the used trapping rate law.

It is noticed that the chemical subsystem is constructed in such a way that the pairs $\left(\mu_{s}, n_{s}\right),\left(\mu_{s}, r_{s}\right)$ and $\left(\operatorname{grad} \mu_{s}, \boldsymbol{j}_{s}\right)$ are related to energies or powers. As a consequence, the existence of the constitutive relations suggests us to search such solutions $n_{\mathrm{c}}$ and $n_{\mathrm{t}}$ that are square integrable in the domain together with their gradients - the square integrability guarantees the finiteness of energy. ${ }^{5}$ In the case of (C.8), we end up in searching for an approximation to its variational formulation from the following subspace of the linear space $\mathcal{C}^{0}([0, d])$ of continuous functions in $[0, d]$ :

$$
\begin{aligned}
S_{\mathrm{h}}^{\mathrm{N}}= & \left\{\chi \in \mathcal{C}^{0}([0, d]): \chi\right. \text { affine in each interval } \\
& {\left[x_{j-1}, x_{j}\right], \text { where }\left\{x_{i}\right\} \text { is a partition of }[0, d] } \\
& \text { such that } 0=x_{0}<x_{1}<\cdots<x_{M}=d, \\
& \text { and } \chi(0)=N\} .
\end{aligned}
$$

(Affine function is a function of the form $f(x)=\alpha x+\beta$.) Since this is also a subspace of an appropriate Sobolev space [30], a relevant problem (the solution of which, if

\footnotetext{
${ }^{5}$ This is the case if there are strictly positive coefficients
} representing the constitutive relations that are bounded below and above [29]. 
smooth enough, satisfies (C.8)) can be set to find $n_{\mathrm{c}} \in S_{\mathrm{h}}^{\mathrm{N}}$ such that

$$
a\left(n_{\mathrm{c}}, \chi\right)=(f, \chi) \quad \text { for all } \chi \in S_{\mathrm{h}}^{0}
$$

where

$$
\begin{aligned}
a(u, v) & =\int_{0}^{d}\left(D \frac{\mathrm{d} u}{\mathrm{~d} x} \frac{\mathrm{d} v}{\mathrm{~d} x}+c u v\right) d x \\
(u, v) & =\int_{0}^{d} u v d x
\end{aligned}
$$

By employing hat functions $\left\{\lambda_{i}\right\}_{i=1}^{M} \subset S_{\mathrm{h}}^{0}, \lambda_{i}\left(x_{j}\right)=\delta_{i j}$ (the Kronecker delta), as a basis for $S_{\mathrm{h}}^{0}$, we can write $n_{\mathrm{c}} \in S_{\mathrm{h}}^{\mathrm{N}}$ by using a finite number of degrees of freedom as $n_{\mathrm{c}}=N \lambda_{0}+\sum_{j=1}^{M} N_{j} \lambda_{j}$, where the hat function $N \lambda_{0}$ is an extension of the boundary value $N$. By using the hat functions $\left\{\lambda_{i}\right\}_{i=1}^{M}$ also as the weight functions $\chi$ in (C.11), we obtain $M$ equations for the unknowns $N_{j}, j=1, \ldots, M$, which are solved at each step of the fixed point iteration procedure. The algorithm goes as

1. Guess $N_{j}$ for $j=1, \ldots, M$,

2. Solve the coefficients for $n_{\mathrm{t}}$ from (C.9),

3. Solve new coefficients $N_{j}$ for $j=1, \ldots, M$, from (C.11),

4. Go to step 2 until converged.

Intuitively, the "fixed point" means that for a given $n_{\mathrm{c}}$, (C.9) results in some $n_{\mathrm{t}}$, and by (C.11) we have $n_{\mathrm{c}}$ again, which must be the same we started from. To prove that the iteration procedure self-consistently converges to a unique fixed point for all initial guesses, it would be sufficient to show that the associated mapping (which maps the array of nodal values $N_{j}$ to new nodal values) is a contraction mapping. This is bypassed here but in practice the procedure does converge.

\section{Dynamic situation}

Parallelling the above strategy, for a given $n_{\mathrm{t}}$, the conduction electron density $n_{\mathrm{c}}$ that belongs to the intersection of (23), (24) and (31) satisfies

$$
\begin{aligned}
\frac{\partial n_{\mathrm{c}}}{\partial t}-\frac{\partial}{\partial x} D \frac{\partial n_{\mathrm{c}}}{\partial x}+c n_{\mathrm{c}} & =f & & \text { in }(0, d) \times(0, T], \\
D \frac{\partial n_{\mathrm{c}}}{\partial x}-k_{\mathrm{ext}}\left(n_{\mathrm{c}}-n_{\mathrm{c}}^{\mathrm{eq}}\right) & =0 & & \text { on }\{0\} \times[0, T], \\
\frac{\partial n_{\mathrm{c}}}{\partial x} & =0 & & \text { on }\{d\} \times[0, T], \\
n_{\mathrm{c}} & =n_{\mathrm{c}}^{\text {ref }} & & \text { on }[0, d] \times\{0\},
\end{aligned}
$$

whereas, for $n_{\mathrm{c}}$ given, the trapped electron density of the intersection satisfies

$$
\begin{aligned}
\frac{\partial n_{\mathrm{t}}}{\partial t}= & -k_{\text {detrap }} n_{\mathrm{t}}+k_{\mathrm{trap}}\left(1-\frac{n_{\mathrm{t}}}{N_{\mathrm{trap}}}\right) n_{\mathrm{c}} \\
& -k_{\mathrm{tb}}\left(n_{\mathrm{t}}-n_{\mathrm{t}}^{\mathrm{eq}}\right) \quad \text { in }[0, d] \times(0, T], \\
n_{\mathrm{t}} & =n_{\mathrm{t}}^{\text {ref }} \quad \text { on }[0, d] \times\{0\} .
\end{aligned}
$$

To solve the problem the finite element method is equipped with time discretisation, and the fixed point iteration procedure is performed at each time step. The initial condition was specified to the steady-state situation of low illumination (approximately $10^{-3}$ sun) by first solving the corresponding steady-state problem for $n_{\mathrm{c}}^{\text {ref }}$ and $n_{\mathrm{t}}^{\mathrm{ref}}$.

This time we employ the function space

$$
\begin{aligned}
S_{\mathrm{h}}= & \left\{\chi \in \mathcal{C}^{0}([0, d]): \quad \chi\right. \text { affine in each interval } \\
& {\left[x_{j-1}, x_{j}\right], \text { where }\left\{x_{i}\right\} \text { is a partition of }[0, d] } \\
& \text { such that } \left.0=x_{0}<x_{1}<\cdots<x_{M}=d\right\}
\end{aligned}
$$

We further employ the time discretisation that $n_{\mathrm{c}}^{k} \in S_{\mathrm{h}}$ (resp. $\left.n_{\mathrm{t}}^{k} \in S_{\mathrm{h}}\right)$ is the approximation of $n_{\mathrm{c}}(t)\left(\right.$ resp. $\left.n_{\mathrm{t}}(t)\right)$ 
at $t=t_{k}=k \Delta t$, where $\triangle t$ is the time step and $k \in \mathbb{N}$.

Then, an approximation to the variational formulation of the time discretised version of (C.14) is defined by the problem: Find $n_{\mathrm{c}}^{k} \in S_{\mathrm{h}}$ recursively for $k>0$ such that

$$
\begin{aligned}
& \left(\triangle t^{-1}\left(n_{\mathrm{c}}^{k}-n_{\mathrm{c}}^{k-1}\right), \chi\right)+a^{k-1 / 2}\left(\frac{1}{2}\left(n_{\mathrm{c}}^{k}+n_{\mathrm{c}}^{k-1}\right), \chi\right) \\
& =b\left(f^{k-1 / 2}, \chi\right) \quad \text { for all } \chi \in S_{\mathrm{h}}, \\
& n_{\mathrm{c}}^{0}=\mathrm{I}_{\mathrm{h}} n_{\mathrm{c}}^{\text {ref }},
\end{aligned}
$$

where

$$
\begin{aligned}
(u, v) & =\int_{0}^{d} u v d x \\
a^{k-1 / 2}(u, v) & =\int_{0}^{d}\left(D \frac{\partial u}{\partial x} \frac{\partial v}{\partial x}+c^{k-1 / 2} u v\right) d x \\
& +k_{\mathrm{ext}} u(0) v(0), \\
b\left(f^{k-1 / 2}, v\right) & =\int_{0}^{d} f^{k-1 / 2} v d x+k_{\mathrm{ext}} n_{\mathrm{c}}^{\mathrm{eq}}(0) v(0),
\end{aligned}
$$

and

$$
\begin{aligned}
& c^{k-1 / 2}=k_{\text {trap }}\left(1-\frac{1}{2 N_{\text {trap }}}\left(n_{\mathrm{t}}^{k}+n_{\mathrm{t}}^{k-1}\right)\right)+k_{\mathrm{cb}}, \quad(\mathrm{C} .21) \\
& f^{k-1 / 2}=g I_{h \nu}\left(t_{k-1 / 2}\right)+k_{\operatorname{detrap}} \frac{1}{2}\left(n_{\mathrm{t}}^{k}+n_{\mathrm{t}}^{k-1}\right)+k_{\mathrm{cb}} n_{\mathrm{c}}^{\mathrm{eq}},
\end{aligned}
$$

and $\mathrm{I}_{\mathrm{h}} n_{\mathrm{c}}^{\mathrm{ref}}$ is the interpolant of $n_{\mathrm{c}}^{\mathrm{ref}} \in \mathcal{C}^{0}([0, d])$ in $S_{\mathrm{h}}$. The same time stepping is used for (C.15), so that $n_{\mathrm{t}}^{k}$ is defined recursively for $k>0$ by

$$
\begin{aligned}
& \triangle t^{-1}\left(n_{\mathrm{t}}^{k}-n_{\mathrm{t}}^{k-1}\right)+\frac{k_{\mathrm{detrap}}}{2}\left(n_{\mathrm{t}}^{k}+n_{\mathrm{t}}^{k-1}\right) \\
& -k_{\mathrm{trap}}\left(1-\frac{1}{2 N_{\mathrm{trap}}}\left(n_{\mathrm{t}}^{k}+n_{\mathrm{t}}^{k-1}\right)\right) \frac{1}{2}\left(n_{\mathrm{c}}^{k}+n_{\mathrm{c}}^{k-1}\right) \\
& +\frac{k_{\mathrm{tb}}}{2}\left(n_{\mathrm{t}}^{k}+n_{\mathrm{t}}^{k-1}\right)-k_{\mathrm{tb}} n_{\mathrm{t}}^{\mathrm{eq}}=0 \\
& n_{\mathrm{t}}^{0}=\mathrm{I}_{\mathrm{h}} n_{\mathrm{t}}^{\mathrm{ref}}
\end{aligned}
$$

The basis for $S_{\mathrm{h}}$ is given by hat functions $\left\{\lambda_{i}\right\}_{i=0}^{M} \subset S_{\mathrm{h}}$, $\lambda_{i}\left(x_{j}\right)=\delta_{i j}$. To obtain finite element equations $n_{\mathrm{c}}^{k}$ and $n_{\mathrm{t}}^{k}$ are expressed in this basis to write (C.17) and (C.23) in matrix form. Finally, the solution procedure can be stated as follows:

1. Set initial conditions $n_{\mathrm{c}}^{0}$ and $n_{\mathrm{t}}^{0}$.

2. Set $k=1$.

3. Solve $n_{\mathrm{c}}^{k}$ and $n_{\mathrm{t}}^{k}$ by fixed point iteration from (C.17) and (C.23).

4. Set $k=k+1$, go to the previous step.

The method is close to the Crank-Nicholson-Galerkin method (see [30]) equipped with the fixed point iteration procedure. Here, however, the bilinear form $a(\cdot, \cdot)$ depends on the time step which is not considered in the analysis of the standard method. We skip further mathematical analysis, and are satisfied with the practical functioning of the algorithm.

\section{References}

1. W. van Roosbroeck, Phys. Rev. 91, 282 (1953)

2. D. L. Scharfetter and H. K. Gummel, IEEE Trans. Electron Devices 16, 65 (1969)

3. G. A. Swartz, J. Appl. Phys. 53, 712 (1982)

4. R. S. Crandall, J. Appl. Phys. 54, 7176 (1983)

5. R. S. Crandall, J. Appl. Phys. 55, 4418 (1984)

6. A. N. Ishaque, J. W. Howard, M. Becker and R. C. Block, J. Appl. Phys. 69, 307 (1991)

7. A. Hagfeldt and M. Grätzel, Chem. Rev. 95, 49 (1995)

8. A. J. Frank, N. Kopidakis and J. van de Lagemaat, Coord. Chem. Rev 248, 1165 (2004) 
9. J. O’M. Bockris and A. K. N. Reddy, Modern Electrochemistry, (New York: Plenum Press, 1970), Vol. 2. p. 694, 696

10. S. R. Morrison, Electrochemistry at Semiconductor and Oxidized Metal Electrodes, (New York: Plenum press, 1980) p. 25

11. P. Atkins and J. de Paula, Physical Chemistry, 8th edn. (Oxford University Press, 2006) p. 98, 795, 952

12. J. O'M. Bockris and A. K. N. Reddy, Modern Electrochemistry, (New York: Plenum Press, 1970), Vol. 1.

13. J. Ferber, R. Stangl and J. Luther, Sol. Energy Mater. Sol. Cells 53, 29 (1998)

14. F. Cao, G. Oskam, G.J. Meyer and P.C. Searson, J. Phys. Chem. 100, 17021 (1996)

15. L. Dloczik, O. Ileperuma, I. Lauermann, L.M. Peter, E.A. Ponomarev, G. Redmond, N.J. Shaw I. and Uhlendorf, J. Phys. Chem. B 101, 10281 (1997)

16. G. Schlichthörl, S.Y. Huang, J. Sprague and A.J. Frank, J. Phys. Chem. B 101, 8141 (1997)

17. L.M. Peter and K.G.U. Wijayantha, Electrochemistry Communications 1, 576 (1999)

18. A.C. Fisher, L.M. Peter, E.A. Ponomarev, A.B. Walker and K.G.U. Wijayantha, J. Phys. Chem. B 104, 949 (2000)

19. A. Kambili, A.B. Walker, F.L. Qiu, A.C. Fisher, A.D. Savin and L.M. Peter, Physica E 14, 203 (2002)

20. F.L. Qiu, A.C. Fisher, A.B. Walker and L.M. Peter, Electrochemistry Communications 5, 711 (2003)

21. M. Grätzel, J. Photochem. Photobiol. C 4, 145 (2003)

22. H. Santa-Nokki, J. Kallioinen, T. Kololuoma, V. Tuboltsev and J.E.I. Korppi-Tommola, J. Photochem. Photobiol. A: Chemistry 182, 187 (2006)

23. I. Montanari, J. Nelson and J.R. Durrant, J. Phys. Chem. B 106, 12203 (2002)
24. J. S. Blakemore, Solid State Physics, 2nd edn. (Cambridge University Press, 1985) p. 379, 394

25. D. Vanmaekelbergh and P.E. de Jongh, J. Phys. Chem. B 103, 747 (1999)

26. J. Bisquert, J. Phys. Chem. B 108, 2323 (2004)

27. J. Bisquert, Phys. Rev. B 77, 235203 (2008)

28. J. Bisquert, A. Zaban and P. Salvador, J. Phys. Chem. B 106, $8774(2002)$

29. A. Bossavit, Computational Electromagnetism: Variational Formulations, Complementarity, Edge Elements, (Academic press series in electromagnetism, 1998) p. 47,48

30. S. Larsson and V. Thomèe, Partial Differential Equations with Numerical Methods, 1st edn. (Texts in applied mathematics, 2003) p. 20, 34, 52, 158

31. J. Bisquert and V.S. Vikhrenko, J. Phys. Chem. B 108, $2313(2004)$

32. J. Kallioinen, M.R. Hassan, G.S. Paraoanu and J.E.I. Korppi-Tommola, J. Photochem. Photobiol. A: Chemistry 195, $352(2008)$ 


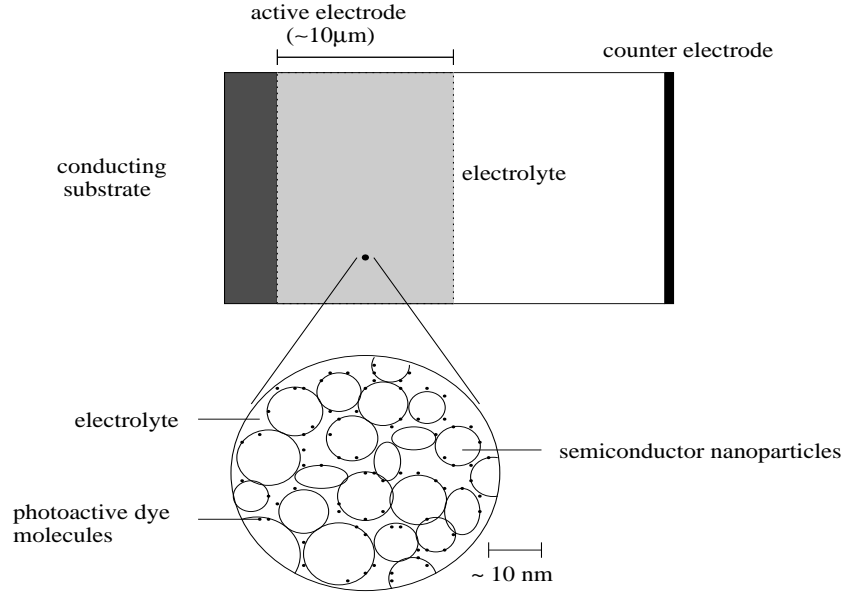

Fig. 1. The schematic structure of the dye cell. The black dot whose enlargement is shown represents a mesoscopic point in the chosen modelling. The enlargement shows a microscopic picture.

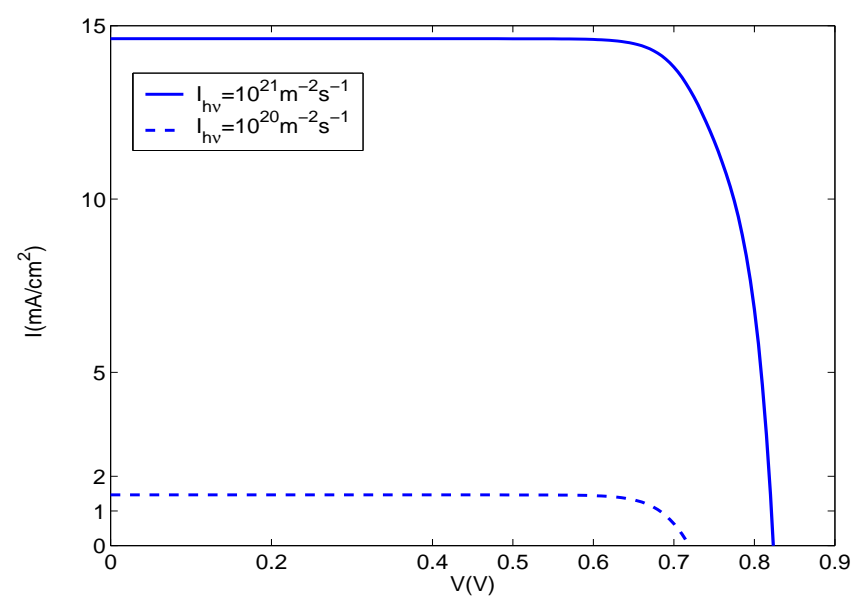

Fig. 2. Simulated current-voltage characteristics under two different illumination levels (the levels correspond to approximately 1 sun and 0.1 sun irradiances).
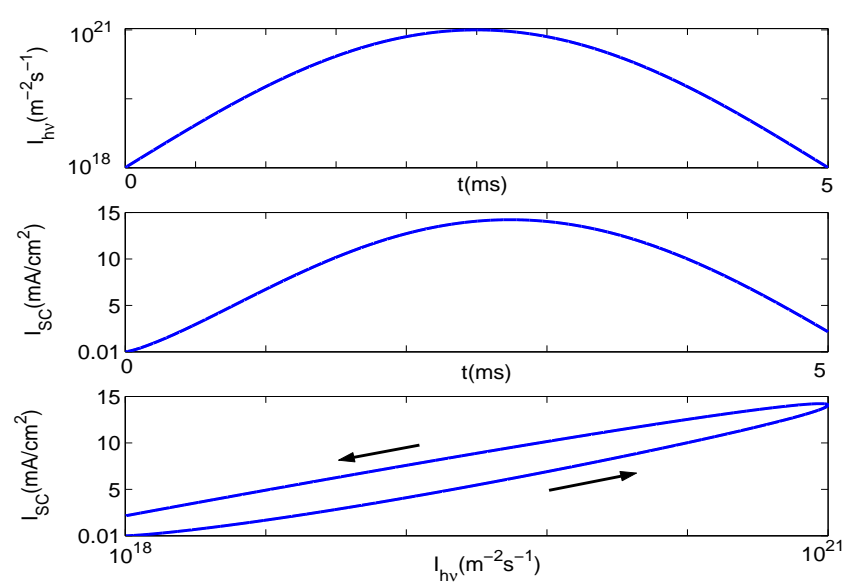

Fig. 3. Short-circuit simulation under fast transient light pulse. Light intensity versus time (top), short-circuit current versus time (center), and the corresponding $\mathrm{I}_{\mathrm{h} \nu} \mathrm{I}_{\mathrm{SC}}$-curve (bottom). The arrows show the direction of time evolution.

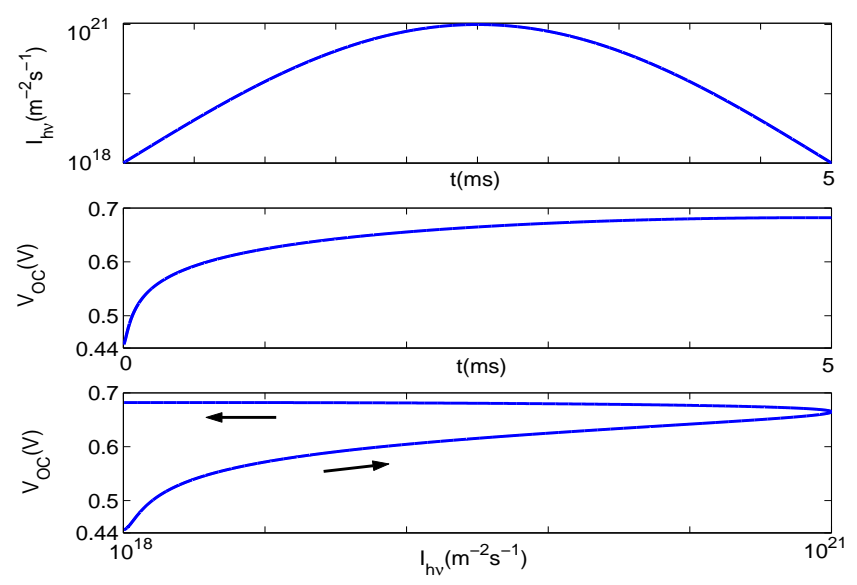

Fig. 4. Open-circuit simulation under fast transient light pulse. Light intensity versus time (top), open-circuit voltage versus time (center), and the corresponding $\mathrm{I}_{\mathrm{h} \nu} \mathrm{V}_{\mathrm{OC}}$-curve (bottom). The arrows show the direction of time evolution. 